\title{
Logarithmic Discrete Wavelet Transform for Medical Image Compression with Diagnostic Quality
}

\author{
M. Shaaban Ibraheem ${ }^{*}$ \\ m-shaaban.ibraheem@lip6.fr \\ Sylvain Hochberg $\ddagger$ \\ sylvain.hochberg@waaves.com
}

Syed Zahid Ahmed*

khalil.hachicha@lip6.fr

\author{
Khalil Hachicha* \\ syed-zahid.ahmed@lip6.fr
}

\author{
Patrick Garda* \\ patrick.garda@lip6.fr
}

\author{
*Sorbonne Universités, UPMC Univ Paris 06, CNRS, LIP6 UMR 7606, 4 Place Jussieu 75005 Paris, France \\ $\ddagger$ CIRA, 11 Rue Charcot, 92800, Puteaux, France
}

\begin{abstract}
This paper presents a novel study of logarithmic discrete wavelet transform (DWT) for medical image compression. It proposes a new technique to compute the DWT using the logarithmic number system(LNS) instead of floating point arithmetic. It investigates its impact on the image quality which is an essential factor for medical images to avoid any misdiagnose. The paper presents detailed experimental results for three medical images modalities: CT, MRI and X-Ray. The results show that the LNS approach gives a significant improvement in the image quality measured with the structural similarity index (SSIM).
\end{abstract}

\section{Categories and Subject Descriptors}

I.4 [Image Processing and computer]: Image processing software, D.2.8 [Metrics] - Performance measures, G.1.2 [Approximation] Wavelets, J.3 [Life and Medical Sciences]: Medical information systems

\section{General Terms}

Performance and Reliability.

\section{Keywords}

Discrete wavelet transform, medical image compression, image quality assessment, logarithmic number system

\section{INTRODUCTION}

Today healthcare systems are spreading into most of the hospitals and medical centers due to the rapid development in the information technology (IT). Medical imaging is considered one of the most important medical information used in telemedicine to provide a remote clinical consultation. Remote consultation requires transferring the data via the network in a fast and efficient way. This becomes a bottleneck in places that have a limited bandwidth and storage capacities. With the continuous increase in the resolution of medical images and number of modalities e.g. Magnetic Resonance Imaging (MRI), X-Ray, Computed Tomography (CT), ultrasound, etc., hospitals require a huge storage capacity. To handle this information, medical image compression is considered as an efficient solution for the limited bandwidth and storage problems. Doctors now can receive the scans not only on their personal computers, but they can also receive it on their tablets or smartphones at urgent cases. Hence the medical image compression represents a big challenge today. Furthermore since medical image contains a sensitive information, it is essential that compressed image preserves the sufficient quality that is essential for radiologists to ensure correct diagnosis.

One of the key advantages of the DWT based compression is that it provides a multi-resolution transform and gives analy sis in spatial frequency and location. Modern image compression algorithms such as JPEG 2000 [1] uses DWT in contrast to the Discrete Cosine Transform (DCT) which is used in JPEG and provide better image quality and compression.

The DWT can be calculated in two ways, the convolution approached which consists of applying filter banks [2] and the lifting scheme approach [3]. Lifting scheme became popular since it requires less memory and is simple due to the symmetry transform making it easier to apply an inverse transform.

According to the literature, the DWT is calculated in floating point (FLP) arithmetic for lossy compression and no work has been published for using the logarithmic number system (LNS) in computing the DWT. The LNS [4] is rarely used since it is not standardized in contrast to the FLP, which is well documented. However LNS is a promising topic for many digital signal processing applications [5]. It has been shown that LNS can give similar and better results in many cases making it an attractive research area for the hardware designers [6].

The main objective of this paper is to investigate computing the DWT in LNS arithmetic, evaluate its impact on the medical image quality and compare the precision of the LNS to FLP. The work presented in this paper according to author's knowledge is the first study in the domain.

The study provided here is a part of the Smart-EEG project [7] that uses WAAVES [8] [9], which is provided by CIRA. WAAVES is a DWT based medical image compression algorithm and is certified for medical uses by radiologists at HEGP hospital [10] in Paris, France. It provides better image quality than JPEG 2000 at the same compression ratio.

The paper is organized as follows: Section 2 provides an overview of the DWT and LNS. Section 3 presents the proposed LNS-DWT. Section 4 reviews the image quality assessment metrics used in this study. Section 5 compares the results obtained by using the 
proposed scheme with the floating point DWT. Finally, conclusion and future work are presented in Section 6.

\section{DWT and LNS OVERVIEW}

\subsection{Lifting DWT}

In this paper, we used the lifting scheme approach due to its efficiency and implementation simplicity. The main idea of the lifting scheme is to decompose a filter bank into a sequence of lifting steps [11]. The filter ployphase matrix is factorized into series of upper and lower triangular matrices called lifting steps. WAAVES and JPEG2000 use the biorthogonal CohenDaubechies-Favreau (CDF) 9/7 filter bank for lossy compression. Figure 1 shows the block diagram of the DWT lifting scheme.

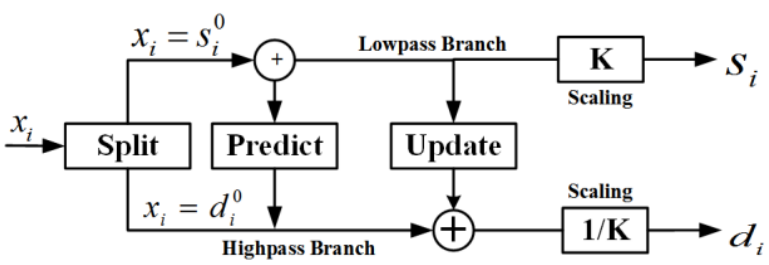

Figure 1. Block diagram of DWT lifting scheme.

The factorized poly phase matrix of CDF 9/7 DWT is defined in (4).

$$
\begin{gathered}
h(z)=h_{e}\left(z^{2}\right)+z^{-1} h_{o}\left(z^{2}\right), \\
g(z)=g_{e}\left(z^{2}\right)+z^{-1} g_{o}\left(z^{2}\right), \\
P(z)=\left[\begin{array}{cc}
h_{e}(z) & h_{o}(z) \\
g_{e}(z) & g_{o}(z)
\end{array}\right], \\
P(z)=\quad\left[\begin{array}{cc}
1 & \alpha\left(1+z^{-1}\right) \\
0 & 1
\end{array}\right]\left[\begin{array}{ccc}
1 & 0 \\
\beta\left(1+z^{-1}\right) & 1
\end{array}\right] \times \\
{\left[\begin{array}{lll}
1 & \gamma\left(1+z^{-1}\right) \\
0 & 1
\end{array}\right]\left[\begin{array}{cc}
1 & 0 \\
\delta\left(1+z^{-1}\right) & 1
\end{array}\right]\left[\begin{array}{ll}
K & 0 \\
0 & \frac{1}{K}
\end{array}\right],}
\end{gathered}
$$

where $h_{e}(z)$ and $g_{e}(z)$ are the even parts and $h_{o}(z)$ and $g_{o}(z)$ are the odd parts of the lowpass $h(z)$ and highpass $g(z)$ filters respectively, and the coefficients values of the filters are:

$$
\begin{array}{ll}
\alpha=-1.586134342, & \beta=-0.05298011854, \\
\gamma=0.8829110762, & \delta=0.4435068522 . \\
K=1.149604398, &
\end{array}
$$

A detailed mathematical description of the lifting scheme algorithm is listed in equations (5)-(12). A signal $x$ is split into odd and even $d_{i}^{n}$ and $s_{i}^{n}$ parts respectively, where $n$ indicates the lifting step number. Each step consists of predict and update, and the last stage is a scaling step:

$$
\text { Odd: } \quad d_{i}^{0}=x_{2 i+1}
$$

Even:

$$
s_{i}^{0}=x_{2 i} \text {, }
$$

Predict 1: $\quad d_{i}^{1}=d_{i}^{0}+\alpha\left(s_{i}^{0}+s_{i+1}^{0}\right)$,

Update 1: $\quad s_{i}^{1}=s_{i}^{0}+\beta\left(d_{i-1}^{1}+d_{i}^{1}\right)$,

Predict 2:

$$
d_{i}^{2}=d_{i}^{1}+\gamma\left(s_{i}^{1}+s_{i+1}^{1}\right)
$$

$$
\begin{aligned}
\text { Update 2: } & s_{i}^{2}=s_{i}^{1}+\delta\left(d_{i-1}^{2}+d_{i}^{2}\right), \\
\text { Odd scaling: } & d_{i}=\frac{d_{i}^{2}}{k}, \\
\text { Even scaling: } & s_{i}=s_{i}^{2} \times k,
\end{aligned}
$$

\subsection{LNS Arithmetic}

Performing computations in LNS requires converting the input data to the logarithmic domain by computing their logarithm. The signed logarithmic format [4] [12] is used in this paper. Assuming that there are two numbers $x$ and $y$ in the floating point format (real world value) and $a$ and $b$ are their logarithmic representation as follows: $i=\log _{2}(|x|), j=\log _{2}(|y|)$ and $a \leq b$.

The basic LNS arithmetic operations of $x$ and $y$ are as follows:

$$
\begin{array}{ll}
\text { Multiplication: } & \log _{2}(x \times y)=a+b \\
\text { Division: } & \log _{2}(x \div y)=a-b \\
\text { Addition: } & \log _{2}(x+y)=b+\log _{2}\left(2^{a-b}+1\right) \\
\text { Subtraction: } & \log _{2}(x-y)=b+\log _{2}\left(2^{a-b}-1\right)
\end{array}
$$

\section{PROPOSED LNS-DWT}

In this section, we explain the classical floating point (FLP) DWT and the proposed LNS-DWT.

\subsection{Classical DWT Scheme}

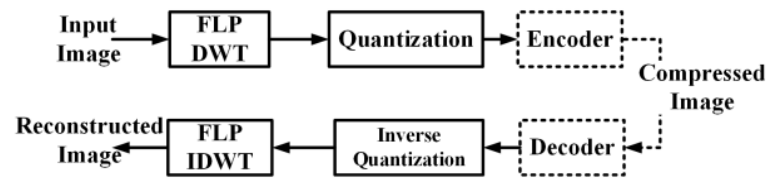

Figure 2. Floating point DWT.

Classical FLP DWT is shown in Figure 2. FLP DWT consists of DWT block and quantization, which is consist of dividing the DWT coefficients by a quantizer value. The quantized samples are then sent to the encoder and formated to create the final bitstream representing the compressed image. On the decoder side the inverse quantization (normalization) is applied to the decoded image samples by multiplying them by the same quantizer value used in the image codec, then inverse discrete transform (IDWT) is calculated.

\subsection{Proposed LNS-DWT Scheme}

The proposed scheme of our work is called LNS-DWT as shown in Figure 3. LNS-DWT is implemented using logarithmic arithmetic by converting all the operations in the lifting scheme represented in (5)-(12) to the logarithmic arithmetic. All the multiplication operations are replaced by addition operations according to (14)

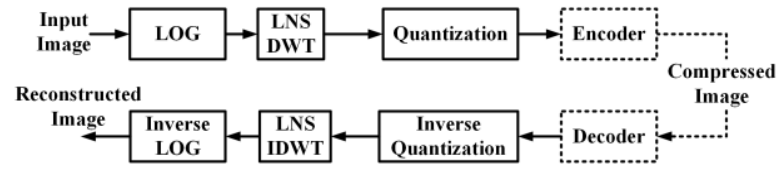

Figure 3. The proposed LNS-DWT. 
and the addition operations are replaced by the functions in (16) and (17).

First, the logarithm operator is applied to the input image then the DWT is calculated in LNS domain. The quantization process is the same as the one used in the classical approach, but instead of dividing the DWT coefficients by the quantizer, the logarithm of the quantizer is subtracted from DWT coefficients, since the division is replaced by subtraction operation according to (15). On the other hand, the decompression process starts by decoding the bitstream, applying the normalization operation, then passing their outputs to the inverse discrete wavelet transform (IDWT). The raw data or reconstructed image raw data is obtained by applying the inverse logarithm to the IDWT outputs.

\section{IMAGE QUALITY ASSESSMENT}

Peak signal-to-noise ratio (PSNR) between the original and reconstructed image as defined in (13) is widely used as an image quality metric, which is function of the mean square error (MSE). It has been shown by researchers that PSNR is not a suitable metric to assess the perceived image quality [13]. Structural similarity index (SSIM) [14] is considered as the more appropriate metric for visual performance, since it measures the image in terms of the human visual system components: structural, luminance and contrast between two images. Due to these reasons radiologists are moving towards considering the SSIM as an alternative to the PSNR metric [15]-[16] for image quality assessment. SSIM is defined in (14).

$$
\begin{gathered}
\operatorname{PSNR}(d B)=\frac{20 \log _{10}(\text { maximum pixel value })}{\sqrt{M S E}}, \\
\operatorname{SSIM}(f, g)=\frac{\left(2 \mu_{f} \mu_{g}+C_{1}\right)+2 \sigma_{f g}+C_{2}}{\left(\mu_{f}^{2} \mu_{g}^{2}+C_{1}\right)\left(\sigma_{f}^{2} \sigma_{g}^{2}+C_{2}\right)}
\end{gathered}
$$

where $f$ and $g$ are the original and reconstructed images, respectively, $\mu_{f}, \mu_{g}$ are the mean intensity values for the two images $f$ and $g$ respectively, $\sigma_{f}, \sigma_{g}$ are the standard deviation of the two images and $C_{1}, C_{2}$ are constants.

\section{EXPERIMENTAL RESULTS}
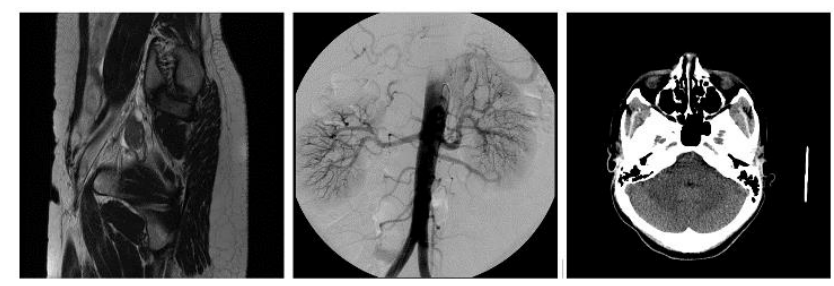

Figure 4. Test samples used in the experiments, from left to right (MRI, X-Ray and CT) scans.

To study the logarithmic DWT, we used three medical images modalities, CT, MRI and X-Ray. Figure 4 shows test samples used in our experiments. The analy sis included calculating the DWT for an input image then quantizing DWT output using different quantizer values. To reconstruct the image, the inverse quantization was applied to the DWT coefficients, then the IDWT was computed. The same process scheme was applied using the LNS. The SSIM and PSNR of the input and reconstructed image was finally calculated for the classical and the proposed scheme.
Figure 6 and Figure 7 show the PSNR and SSIM values for different quantization step values. Observe that the quality is degraded with increasing quantization step value. As it is known, a higher quantization step gives a better compression ratio and less image quality.

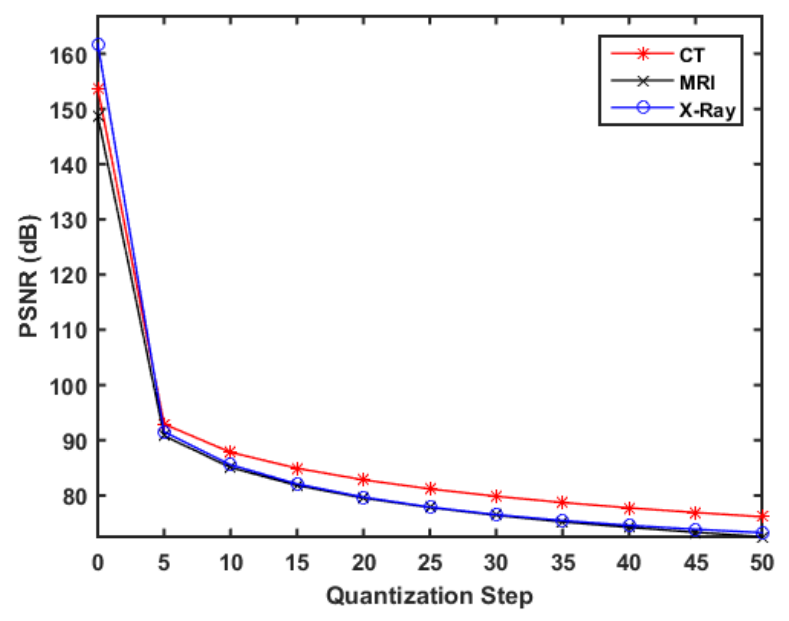

Figure 5. PSNR vs Quantization Step for FLP DWT.

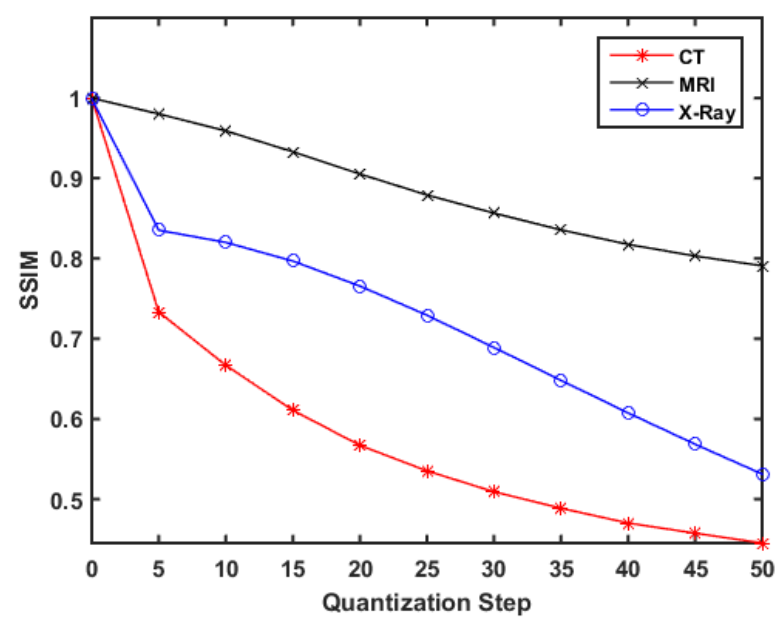

Figure 6. SS IM vs Quantization Step for FLP DWT.

The experiments were conducted again but using LNS-DWT scheme. Figure 8 and Figure 9 show the impact on the PSNR and SSIM values when varying the number of the decimal digits of the fraction part in the LNS-DWT output that was kept after the quantization. While in the classical scheme, we discarded all the decimal fraction, in this scheme we kept at least two decimal digits to obtain SSIM greater than 0.9 which gave an acceptable image quality required by radiologists.

Keeping the fraction part was very important because a slight change in the data in the logarithm domain would lead to a significant effect on the accuracy when switching back to the floating point domain. Since the quantization process was converted to a subtraction operation in the logarithmic domain, so changing the quantization step did not give a significant impact on the SSIM. Hence we used the number of decimal digits of fraction part which made it a new factor that affected the quality. 


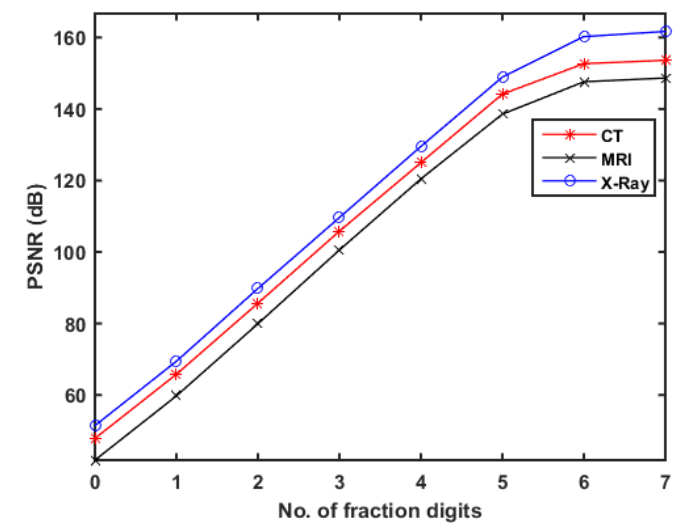

Figure 7. PSNR vs Number of the fraction digits for the proposed LNS-DWT.

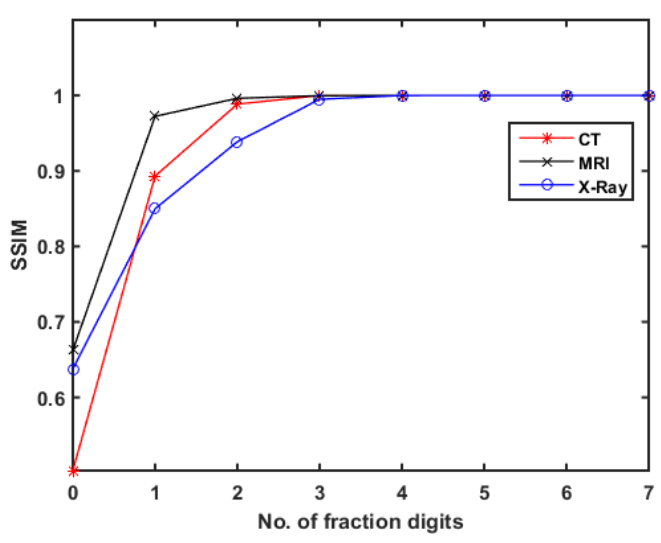

Figure 8. SS IM vs Number of the fraction digits for the proposed LNS-DWT.

\section{CONCLUSION}

A new study was presented in this paper. The study introduced a novel technique to calculate the DWT by using logarithmic arithmetic. Different experiments were conducted on different medical image modalities. The experimental results showed that the LNS-DWT gave better image quality. The proposed LNS-DWT raises a new challenge on the encoder side regarding how theses LNS values could be encoded. Future works include further refining and investigating LNS results for the different modalities., and also, developing a new encoder capable to handle both fractional and integer parts of quantized data.

\section{ACKNOWLEDGMENTS}

The authors thank Didier Heudes and Fayez Kaddhou from HEGP for their help and all the members of the Smart-EEG project for fruitful exchanges. This work was supported by a research CIFRE contract between ANRT, UPMC and CIRA. It also benefited of the support of the Ile-de-France region in the framework of the SmartEEG FUI project. Finally this work fits in the scope of the SM ART Labex, which is supported by French state funds managed by the ANR within the Investissements d'Avenir programme under reference ANR-11-IDEX-0004-02.

\section{REFERENCES}

[1] Ahmed, S.Z. et al. 2014. SmartEEG: A multimodal tool for EEG signals. 2014 IEEE Faible Tension Faible Consommation (FTFC) (May 2014), 1-4.
[2] Coleman, J.N. et al. 2000. Arithmetic on the European logarithmic microprocessor. IEEE Transactions on Computers. 49, 7 (Jul. 2000), 702-715.

[3] Collange, S. et al. 2006. Floating Point or LNS: Choosing the Right Arithmetic on an Aapplication Basis. 9th EUROMICRO Conference on Digital System Design: Architectures, Methods and Tools, 2006. DSD 2006 (2006), 197-203.

[4] Daubechies, I. and Sweldens, W. 1998. Factoring wavelet transforms into lifting steps. Journal of Fourier Analysis and Applications. 4, 3 (May 1998), 247-269.

[5] Fu, H. et al. 2007. OptimizingLogarithmic Arithmetic on FPGAs. 15th Annual IEEE Symposium on FieldProgrammable Custom Computing Machines, 2007. FCCM 2007 (Apr. 2007), 163-172.

[6] Kowalik-Urbaniak, I. et al. 2014. The quest for "diagnostically lossless" medical image compression: a comparative study of objective quality metrics for compressed medical images. (2014), 903717-903717-16.

[7] Mallat, S.G. 1989. A theory for multiresolution signal decomposition: the wavelet representation. IEEE Transactions on Pattern Analysis and Machine Intelligence. 11, 7 (Jul. 1989), 674-693.

[8] Mhedhbi, I. et al. 2014. Mask motion adaptive medical image coding. 2014 IEEE-EMBS International Conference on Biomedical and Health Informatics (BHI) (Jun. 2014), 408-411.

[9] Mhedhbi, I. et al. 2013. Towards a Mobile Implementation of Waaves for Certified Medical Image Compression in EHealth Applications. Wireless Mobile Communication and Healthcare. B. Godara and K.S. Nikita, eds. Springer Berlin Heidelberg. 79-87.

[10] Sriraam, N. and Shy amsunder, R. 2011. 3-D medical image compression using 3-D wavelet coders. Digital Signal Processing. 21, 1 (Jan. 2011), 100-109.

[11] Swartzlander, E.E. et al. 1983. Sign/Logarithm Arithmetic for FFT Implementation. IEEE Transactions on Computers. C-32, 6 (Jun. 1983), 526-534.

[12] Sweldens, W. 1998. The Lifting Scheme: A Construction of Second Generation Wavelets. SIAM Journal on Mathematical Analysis. 29, 2 (Mar. 1998), 511-546.

[13] Taubman, D.S. and Marcellin, M.W. 2002. JPEG2000 Image Compression Fundamentals, Standards and Practice. Springer US.

[14] Wang, Z. et al. 2004. Image quality assessment: from error visibility to structural similarity. IEEE Transactions on Image Processing. 13, 4 (Apr. 2004), 600-612.

[15] Wang, Z. and Bovik, A.C. 2009. Mean squared error: Love it or leave it? A new look at Signal Fidelity Measures. IEEE Signal Processing Magazine. 26, 1 (Jan. 2009), 98-117.

[16] Hôpital européen Georges-Pompidou. Hôpital européen Georges-Pompid 